Revista de Psicología Vol. 30 (1), 2012 (ISSN 0254-9247)

\title{
Razones para vivir en jóvenes adultos: validación del RFL-YA
}

\author{
Mónica Cassaretto B. ${ }^{1}$ y Patricia Martínez U. ${ }^{2}$ \\ Pontificia Universidad Católica del Perú
}

Se analizan las propiedades psicométricas del Inventario Razones para Vivir, versión para jóvenes adultos (RFL-YA) en 368 estudiantes universitarios de Lima, Perú, de ambos sexos, cuyas edades oscilan entre los 16 y 30 años. Se aplicó el RFL-YA de Gutiérrez et al. (2002), además de la Escala de Sentido de Coherencia (SOC) de Antonovsky (1993) y la Escala de Esperanza de Herth (Arnau, Martínez, Niño de Guzmán, Herth \& Yoshiyuki, 2010) para determinar la validez convergente de la escala. Los resultados mostraron elevados índices de confiabilidad. Un coeficiente de 0.93 para la prueba total y coeficientes entre 0.83 y 0.92 para cada una de las áreas de la prueba. La estructura factorial resultó adecuada, se reprodujeron los 5 factores con un $63.74 \%$ de varianza explicada. La correlación con las escalas de Sentido de Coherencia y Esperanza resultaron positivas, altas y significativas, con lo que se confirma la validez y confiabilidad del inventario para poblaciones similares a las del presente estudio. Palabras clave: razones para vivir, esperanza, sentido de coherencia.

\section{Reasons for living in young adults: Validation of the RFL-YA}

The study analyses the psychometric properties of the Reasons for Living Inventory for Young Adults (RFL-YA) in a group of 368 university students from Lima, Peru, from both sexes and whose ages fluctuate between 16 and 30 years of age. The instruments used were the RFL-YA (Gutiérrez et al., 2002), Antonovsky's Sense of Coherence Scale (SOC) (1993) and Hert's Hope Scale (Arnau, Martínez, Niño de Guzmán, Herth \& Yoshiyuki, 2010) in order to determine the convergent validity of the RFL-YA. Results show elevated reliability indexes. A 0.93 coefficient for the whole test and a 0.83 and 0.92 for each of the areas of the test. The factorial structure is adequate, and all 5 factors were reproduced with a $63.74 \%$ of explained variance. The correlation between the Sense of Coherence Scale and the Hope Scale resulted positive, high and significant, thus confirming the validity and reliability of the inventory for similar populations.

Key words: Sense of coherence, reasons for living, hope.

1 Magíster en Psicología Clínica y de la Salud de la Universidad Nacional Mayor de San Marcos. Profesora Asociada del Departamento de Psicología de la PUCP. Contacto: Av. Universitaria 1801, Lima 32, Perú; mcassar@pucp.edu.pe

2 Doctora en Psicología de la Universidad Autónoma de Barcelona, España. Profesora Principal del Departamento de Psicología de la PUCP. Contacto: Av. Universitaria 1801, Lima 32, Perú; pmartin@pucp.edu.pe 

El suicidio ha sido objeto de numerosas investigaciones a nivel mundial debido a que es considerado uno de los mayores problemas de salud pública por la Organización Mundial de la Salud (OMS). Así, el último reporte mundial de salud mental lo ubica dentro de las 15 principales causas de mortandad en distintas etapas de la vida. Esta situación es más preocupante en población joven, ya que el suicidio es la tercera causa de muerte para la población entre los 15 y 34 años de edad (OMS, 2001).

En el Perú, según los estudios realizados por el Instituto Nacional de Salud Mental "Honorio Delgado-Hideyo Noguchi" la prevalencia de intento de suicidio en Lima Metropolitana es de 5.2\% (INSM, 2002). De manera similar a lo hallado a nivel mundial, en un estudio que reportaba las características del total de pacientes atendidos en un hospital general de Lima en el período 1995-2004, se encontró que la mayoría eran adolescentes y adultos tempranos, hallándose que el $74 \%$ de dichos pacientes tenían entre 15 y 29 años de edad (Paredes, Orbegoso \& Rosales, 2006).

Los especialistas en suicidio consideran que este debe ser visto desde un continuo, que se inicia con la aparición de pensamientos sobre el mismo y que puede progresivamente culminar en su realización. Por ello, una forma de evaluar el riesgo de suicidio implica la evaluación de la ideación suicida. A ello se debe agregar que se trata de un fenómeno complejo que puede ser explicado por la interacción de múltiples factores, entre los que se encuentran los biológicos, psicosociales y ambientales (Westefeld et al., 2000).

Entre los factores psicosociales asociados al riesgo suicida se encuentra la presencia de sintomatología depresiva, sentimientos de desesperanza, pensamientos de indefensión, baja autoestima y pobres habilidades de afrontamiento. A nivel cognitivo, se halla que las personas con alta ideación suicida presentan un locus de control externo, 
menores habilidades en la búsqueda de solución de problemas, dificultades en la capacidad para recuperar recuerdos positivos en la memoria autobiográfica y actitudes diferentes ante la vida y la muerte (Britton et al., 2008; Dogra, Basu \& Das, 2011; Edwards \& Holden, 2003; Gutiérrez et al., 2002; Wang, Lightsey, Pietruszka, Uruk \& Wells, 2007; Westefeld et al., 2000).

Como se puede observar, la mayor parte de la investigación se ha concentrado en el estudio de los factores de riesgo que predisponen a la realización de un comportamiento suicida. En proporción a esto, muy poco se ha estudiado sobre los recursos adaptativos presentes en las personas no suicidas (Rosero, 2008; Wang et al., 2007). Asimismo, Osman et al. (1998) consideran que centrarse en la evaluación de los factores de riesgo suicida no ayuda de forma completa a la comprensión y prevención del riesgo al suicidio; por ello, promueven el estudio de los factores de riesgo y de los factores protectores en simultáneo.

Uno de los factores protectores más potentes contra los pensamientos y acciones suicidas son las creencias que tienen las personas que prefieren vivir a cometer suicidio cuando enfrentan situaciones de adversidad. Los estudios iniciados en esa línea por Linehan, Goodstein, Nielsen y Chiles (1983) han llevado a establecer que son las creencias y expectativas asociadas a la vida las que pueden mitigar la inclinación a la conducta suicida, ya que aquellas personas con fuertes razones para vivir son más propensas a resistir el estrés y la desesperanza, a no considerar el suicidio y, por tanto, a no llevarlo a cabo. En cambio, los individuos que carecen de creencias positivas o altas expectativas por la vida y tienen menos preocupaciones sobre las consecuencias de su potencial suicidio en su entorno social están más propensos a presentar conductas suicidas.

Marsha Linehan, junto a sus colaboradores (1983), realizó varios estudios que llevaron a la creación de un instrumento para evaluar el riesgo suicida, asumiendo que lo que diferencia a los individuos suicidas de los no suicidas es el sistema de creencias que los motiva a vivir. Para ello, crearon una prueba llamada Inventario Razones para Vivir (Reasons for Living Inventory, RFL), conformada por 48 ítems que 
evalúan las creencias para no cometer suicidio. Estas creencias se organizan en 6 subescalas: creencias de supervivencia y fortaleza, creencia de responsabilidad familiar, preocupaciones concernientes a los hijos, el miedo al suicidio, el miedo a la desaprobación social y las objeciones morales. Desde su creación, el RFL se mostró como un excelente instrumento para evaluar el riesgo suicida; además, existen distintos estudios que citan sus propiedades psicométricas al señalar altos niveles de consistencia interna y validez de constructo en muestras de adolescentes delincuentes, estudiantes saludables y adultos psiquiátricos. El RFL también ha demostrado validez divergente con otras medidas de comportamiento e ideación suicida, depresión y desesperanza (Gutiérrez et al., 2002; Linehan et al., 1983; Westefeld et al., 2000).

A partir del reconocimiento de que las razones para vivir pueden ser ligeramente distintas de acuerdo a la etapa de desarrollo en que se encuentra la persona, es que se han empezado a crear distintas versiones del instrumento. Así, tenemos una primera versión del RFL llevada a cabo por Westefeld et al. (2000) dirigida a estudiantes universitarios (CS-RFL, College Student Reasons for Living Inventory).

Además, existe una versión resumida del inventario llamada RFL-B (Reasons for Living Inventory Brief), desarrollada por Ivanoff, Jang, Smyth y Linehan en 1994, que recoge 12 de los ítems originales del RFL. Esta versión ha mostrado una adecuada consistencia interna y validez divergente con medidas de depresión y desesperanza (Ivanoff et al., como se cita en Westefeld et al., 2000).

Posteriormente, Osman et al. (1998) desarrollaron una versión de la prueba para adolescentes entre los 14 y 18 años (RFL-A, Reasons for Living Inventory for Adolescents). Esta escala consta de 32 ítems agrupados en cinco factores: optimismo hacia el futuro, preocupaciones relacionadas con el suicidio, alianza familiar, aceptación o apoyo de pares, y autoaceptación. En su estudio, los autores confirmaron la presencia de los 5 factores además de relaciones significativas con escalas de desesperanza, psicopatología, probabilidad de suicidio y conductas suicidas.

Oquendo et al. (2000) adaptaron la escala RFL al español en población latina norteamericana. Evaluaron su estructura factorial, 
confiabilidad, validez de contenido y de constructo en una muestra clínica de individuos con intento de suicidio. Esta versión mostró adecuadas propiedades psicométricas, una estructura factorial de 6 dimensiones y una elevada consistencia interna y temporal. Asimismo, se encontraron diferencias significativas en las puntuaciones del RFL entre individuos con riesgo de suicidio alto y bajo, definido clínicamente. De forma similar se validó el instrumento con personas con conducta suicida en Colombia obteniéndose resultados similares (Valencia et al., 2009).

Edelstein et al. desarrollaron en el 2009 una versión de la prueba para adultos tardíos llamada RFL-OA (Reasons for Living-Older Adult Scale), que fue probada en dos estudios con muestras no clínicas y una muestra clínica de pacientes adultos tardíos que sufrían de depresión, ideación suicida y presentaban bajos niveles de salud física y mental. Los resultados mostraron que el RFL-OA presentaba altos niveles de consistencia interna con fuerte evidencia de validez convergente, discriminante y de criterio (Edelstein et al., 2009).

En el 2002 Gutiérrez et al. decidieron crear una nueva versión del RFL para adultos jóvenes, al encontrar que la versión para estudiantes universitarios CS-RFL (Westefeld et al., 2000) presentaba dos limitaciones importantes para su uso. Primero, debido a su gran similitud con la escala original no permitía extraer todas las razones importantes para vivir en este grupo en específico. Segundo, el número extenso de ítems (60) dificultaba la aplicación rápida de la escala junto con otros instrumentos al hacer investigación con esa población.

Así, Gutiérrez et al. (2002) desarrollaron una medida alternativa para evaluar las razones para vivir en jóvenes adultos, el RFL-YA (Reasons for Living for Young Adults), demostrando en tres estudios con universitarios norteamericanos ser una medida más sólida y parsimoniosa que las versiones anteriores.

El RFL-YA se construyó a partir del CS-RFL y del RFL-A. Para ello, se seleccionó un conjunto de ítems de estas versiones, se analizó la consistencia interna y la estructura factorial de la escala, con lo que se eliminaron algunos ítems y se modificaron ligeramente otros. 
Esto dio como resultado la escala final de 32 ítems organizados en cinco dimensiones: relaciones familiares, relaciones con pares, creencias de afrontamiento, expectativas frente al futuro y autoevaluación positiva. Al aplicarse a distintas muestras, el instrumento mostró adecuadas propiedades psicométricas. Además de una estructura factorial sólida y una elevada consistencia interna, se hallaron correlaciones importantes con las escalas de Probabilidad de Suicidio (SPS) y de Ideación Suicida Positiva y Negativa (PANSI), así como con la Escala de Desesperanza de Beck y la Escala de Depresión, Ansiedad y Estrés (DASS).

En Latinoamérica, existe un estudio de validación del RFL-YA llevado a cabo en Colombia por Rosero (2008) con una muestra de estudiantes universitarios de la ciudad de San Juan de Pasto. En ese estudio, la autora comparó la versión traducida y una versión más amplia del inventario. Los resultados mostraron que la versión traducida del inventario era superior a la ampliada, con un $60.2 \%$ de varianza explicada y una consistencia interna para la prueba total de .92. Encontró relaciones significativas del RFL-YA con medidas de autoestima, resiliencia ante el suicidio, ideación suicida, desesperanza y depresión. Además, la prueba demostró capacidad para discriminar entre muestras de adultos jóvenes con y sin conductas suicidas.

De los estudios mencionados previamente se desprende la necesidad de estudiar las propiedades psicométricas del RFL-YA en una población adulta temprana de Lima, Perú, particularmente debido a las altas tasas de suicidio en este grupo (OMS, 2001). Por ello, es importante constatar si también aquí el inventario cumple con las condiciones necesarias para su utilización. Para conseguir este objetivo, se analiza la estructura factorial del instrumento y la confiabilidad de la prueba total y cada una de sus escalas. Asimismo, con el fin de determinar la validez convergente, se analiza su correlación con otras variables como esperanza y sentido de coherencia, con las que se espera una correlación positiva y significativa. 


\section{Método}

\section{Participantes}

En el estudio participaron un total de 368 jóvenes universitarios de ambos sexos, de una universidad privada de Lima, Perú. De ellos, $201(54.6 \%)$ participantes fueron varones y 167 (45.4\%) mujeres. El rango de edad se ubicó entre los 16 y los 30 años, con una edad promedio de 19.7 años. El 58.2\% $(n=214)$ se encontraba cursando carreras de letras y el $41.9 \%(n=154)$, carreras de ciencias.

\section{Instrumentos}

Escala de Razones para Vivir para Jóvenes Adultos (RFL-YA) (Gutiérrez et al., 2002)

Esta escala está diseñada para poblaciones entre los 17 y los 30 años. Es una escala tipo Likert con 32 ítems y 6 opciones de respuesta que van de "No es una razón importante" a "Es una razón sumamente importante". Incluye 5 escalas o factores: relaciones familiares (FR), relaciones con pares (PR), creencias de afrontamiento (CB), expectativas hacia el futuro (FE) y autoevaluación positiva (PSE). Los estudios realizados con la escala han demostrado buenas propiedades psicométricas en población norteamericana y colombiana (Gutiérrez et al., 2002; Rosero, 2008).

Escala de Esperanza Revisada de Herth-HHS-R (Martínez, Cassaretto \& Herth, 2011)

La medición de la esperanza se realizó a través de la versión en español de esta escala, que consta de 28 ítems con 4 opciones de respuesta que van de "Con frecuencia" a "Nunca". La versión original en español fue validada por Arnau et al. (2010). En ambos estudios la prueba mostró elevados índices de confiabilidad y una estructura factorial consistente con estudios previos. En el presente estudio se aplicó un alfa de Cronbach con el fin de determinar la consistencia interna del instrumento. Se obtuvo un coeficiente de .89 . 
Cuestionario de Orientación hacia la Vida (SOC) (Antonovsky, 1993)

Se utilizó para evaluar el sentido de coherencia, Este cuestionario consta de 29 ítems con una escala tipo Likert del 1 al 7. Cada ítem incluye diferentes criterios en los extremos. Por ejemplo, 1 puede ser "Nunca tuve este sentimiento", mientras que el 7 sería "Siempre tuve este sentimiento".

Antonovsky (1993) reportó un índice de consistencia interna de 0.82 para la prueba total. Estos resultados se han confirmado en numerosas investigaciones, sin embargo, han surgido controversias con respecto a las áreas de la misma (Von Bothmer \& Fridlund, 2003). Asimismo, se han llevado a cabo estudios sobre la validez convergente del instrumento con medidas de depresión, ansiedad y estrés con resultados muy consistentes (Frenz, Carey \& Jorgensen, como se cita en Von Bothmer \& Fridlund, 2003). En el Perú se han realizado estudios con la prueba que van en el mismo sentido de los descritos anteriormente (Arévalo, 2007; Saravia, 2010).

En el presente estudio se llevó a cabo un análisis de la confiabilidad interna del cuestionario y se obtuvo un coeficiente alfa de .91.

\section{Procedimiento}

Esta investigación, al ser de tipo psicométrica, se realizó en una sola aplicación en las aulas de clase. Los sujetos que participaron en esta investigación firmaron un consentimiento informado que les indicaba las condiciones de investigación y sus derechos como participantes de la misma.

\section{Resultados}

Los resultados del análisis de la estructura factorial del instrumento, a través del análisis de componentes principales con rotación oblimin y 5 factores, obtuvo un KMO de .91 $(p<.001)$. La varianza total explicada alcanzó un $63.74 \%$. 


\section{Tabla 1}

Análisis Factorial de RFL-YA

\begin{tabular}{|c|c|c|c|c|c|}
\hline Ítems & $F R$ & $F E$ & $P R$ & $C B$ & PSE \\
\hline 7 & .83 & & & & \\
\hline 9 & .84 & & & & \\
\hline 12 & .80 & & & & \\
\hline 13 & .82 & & & & \\
\hline 21 & .82 & & & & \\
\hline 24 & .79 & & & & \\
\hline 26 & .87 & & & & \\
\hline 1 & & .65 & & & \\
\hline 3 & & .64 & & & \\
\hline 8 & & .82 & & & \\
\hline 15 & & .82 & & & \\
\hline 25 & & .79 & & & \\
\hline 29 & & .75 & & & \\
\hline 31 & & .81 & & & \\
\hline 5 & & & .82 & & \\
\hline 10 & & & .81 & & \\
\hline 14 & & & .83 & & \\
\hline 16 & & & .86 & & \\
\hline 17 & & & .86 & & \\
\hline 30 & & & .83 & & \\
\hline 2 & & & & .59 & \\
\hline 6 & & & & .68 & \\
\hline 11 & & & & .76 & \\
\hline 22 & & & & .77 & \\
\hline 27 & & & & .70 & \\
\hline 28 & & & & .82 & \\
\hline 32 & & & & .55 & \\
\hline 4 & & & & & .68 \\
\hline 18 & & & & & .86 \\
\hline 19 & & & & & .71 \\
\hline 20 & & & & & .76 \\
\hline \multirow[t]{2}{*}{23} & & & & & .86 \\
\hline & $33.14 \%$ & $10.97 \%$ & $6.99 \%$ & $6.43 \%$ & $6.19 \%$ \\
\hline
\end{tabular}


Todos los ítems se ubicaron en el factor que les corresponde con una carga factorial superior al 0.40 , lo que indica una estructura factorial consistente con los estudios previos de la escala. Luego se analizó la correlación entre los diferentes factores de la prueba, que se presentan en la Tabla 2. Como se observa, todas las dimensiones del instrumento presentan una correlación positiva y significativa $(p<.001)$ entre sí. Las correlaciones más altas se encuentran entre las escalas de relaciones familiares (FR) y relación con pares (PR), seguida por expectativas hacia el futuro $(\mathrm{FE})$ y creencias de afrontamiento $(\mathrm{CB})$.

\section{Tabla 2}

Correlaciones entre los factores del RFL-YA

\begin{tabular}{ccccl}
\hline Escalas & $F E$ & $P R$ & $C B$ & $P S E$ \\
\hline FR & $.27^{* * *}$ & $.45^{* * *}$ & $.27^{* * *}$ & $.28^{* * *}$ \\
FE & & $.24^{* * *}$ & $.35^{* * *}$ & $.29^{* * *}$ \\
PR & & & $.23^{* * *}$ & $.30^{* * *}$ \\
CB & & & & $.29^{* * *}$ \\
\hline
\end{tabular}

${ }^{* * *} p<.001$

Luego, con el fin de evaluar la confiabilidad de la prueba total y de cada una de las escalas que la componen, se realizó un alfa de Cronbach, que mostró una elevada consistencia interna en todas las escalas (Tabla 3). La prueba total en conjunto obtuvo un coeficiente de 93. 


\section{Tabla 3}

Confiabilidad de las áreas del RFL-YA

\begin{tabular}{cccccccccc}
\hline Items & $r$ & Items & $r$ & Items & $r$ & Items & $r$ & Items & $r$ \\
\hline 7 & .73 & 1 & .55 & 5 & .73 & 2 & .44 & 4 & .58 \\
9 & .77 & 3 & .59 & 10 & .72 & 6 & .58 & 18 & .74 \\
12 & .74 & 8 & .74 & 14 & .77 & 11 & .62 & 19 & .59 \\
13 & .75 & 15 & .76 & 16 & .79 & 22 & .63 & 20 & .65 \\
21 & .76 & 25 & .72 & 17 & .79 & 27 & .57 & 23 & .75 \\
24 & .74 & 29 & .62 & 30 & .76 & 28 & .71 & & \\
26 & .81 & 31 & .70 & & & 32 & .48 & & \\
\hline Coef. & .92 & Coef. & .88 & Coef. & .91 & Coef. & .83 & Coef. & .85 \\
\hline
\end{tabular}

Por otro lado, con el fin de determinar la validez convergente de la escala, se analizaron las correlaciones con la Escala de Sentido de Coherencia (SOC) y la Escala de Esperanza (HHS-R).

\section{Tabla 4}

Correlaciones con otros instrumentos

\begin{tabular}{llc}
\hline & SOC & $H H S-R$ \\
\hline RFL-YA Total & $.56^{* * *}$ & $.59^{* * *}$ \\
Relaciones familiares (FR) & $.42^{* * *}$ & $.43^{* * *}$ \\
Expectativas hacia el futuro (FE) & $.36^{* * *}$ & $.44^{* * *}$ \\
Relaciones entre pares (PR) & $.40^{* * *}$ & $.41^{* * *}$ \\
Creencias de afrontamiento (CB) & $.37^{* * *}$ & $.39^{* * *}$ \\
Autoevaluación positiva (PSE) & $.52^{* * *}$ & $.51^{* * *}$ \\
\hline
\end{tabular}

${ }^{* * *} p<.001$

En la Tabla 4 se puede observar que tanto la prueba en conjunto como cada una de sus escalas correlacionan positiva y significativamente con sentido de coherencia y esperanza, como se esperaría teóricamente. Las correlaciones más altas se presentan con la prueba total. La escala 
de autoevaluación positiva es la que mayor correlación presenta con ambos instrumentos.

Finalmente, en la Tabla 5 se presentan los resultados descriptivos del RFL-YA en la población estudiada. El puntaje bruto promedio para la prueba total fue de $153.95(D E=22.09)$.

\section{Tabla 5}

Descriptivos del RFL-YA

\begin{tabular}{lccccc}
\hline & No. de items & Minimo & Máximo & $M$ & DE \\
\hline Relaciones familiares (FR) & 7 & 1.29 & 6 & 4.75 & 1.03 \\
Expectativas hacia el futuro (FE) & 7 & 1.43 & 6 & 5.01 & 0.84 \\
Relaciones entre pares (PR) & 6 & 1 & 6 & 4.53 & 1.03 \\
Creencias de afrontamiento (CB) & 7 & 1.86 & 6 & 4.82 & 0.89 \\
Autoevaluación positiva (PSE) & 5 & 1.80 & 6 & 4.93 & 0.94 \\
\hline
\end{tabular}

\section{Discusión}

El RFL-YA es un instrumento que presenta una serie de ventajas: su aplicación demanda poco tiempo, sus enunciados evalúan el riesgo suicida de manera indirecta siendo menos susceptible a la deseabilidad social, resulta un instrumento útil para el desarrollo de campañas de promoción y prevención en salud mental, así como para investigación con esta población. Si bien el RFL-YA evalúa riesgo suicida, tiene la ventaja de analizar los recursos con los que cuenta el individuo, exponiendo factores protectores y no solo los de riesgo.

Los hallazgos de esta investigación resultan prometedores ya que el RFL-YA muestra, en el grupo estudiado, niveles de consistencia interna bastante altos y evidencia de validez de constructo y convergente con otros instrumentos. Así, encontramos que la estructura factorial hallada es bastante sólida y está conformada por cinco factores que explican el $63.74 \%$ de la varianza de la prueba. Por otro lado, las cargas factoriales de los ítems son superiores al .55 por lo que se consideran bastante altos, 
superando el punto recomendado de .40. Por otro lado, al observar las áreas que emergen del análisis factorial encontramos que las áreas que aparecen corresponden claramente a las planteadas por los autores (Gutiérrez et al., 2002) y estas son, en orden de aparición: relaciones familiares, expectativas frente al futuro, relaciones con pares, creencias de afrontamiento, y autoevaluación positiva; todo lo cual apoya la validez de constructo de esta prueba.

Los coeficientes de confiabilidad obtenidos a través de los índices de consistencia interna alfa de Cronbach se ubican entre .83 y .92 que, de acuerdo a los criterios de George y Mallery (2001) resultan entre altos y excelentes. Estos índices se pueden sustentar en la elevada capacidad discriminativa de los ítems, los cuales obtienen correlaciones ítem-test superiores al .55 (Jong, 2004). Cabe mencionar que puntajes similares se hallaron en otros estudios en población joven (Gutiérrez et al., 2002; Muehlenkamp, Gutiérrez, Osman \& Barrios, 2005; Wang et al., 2007). El que los coeficientes de confiabilidad de las áreas de la prueba sean altos apoya la idea de que los ítems evalúan un mismo constructo y están interrelacionados. Por tanto, podemos afirmar que esta prueba presenta alta consistencia y coherencia interna.

En el análisis de medias hallamos que el área en la cual se presentaron los puntajes más altos fue expectativas frente al futuro, la cual emerge como la principal razón para vivir; esta razón indica la creencia de que los planes y metas se realizarán, y que el futuro traerá cosas buenas en la vida. El que los estudiantes manifiesten a este como la principal razón para vivir es coherente con la etapa de desarrollo en que se encuentran, ya que la adultez temprana es el período en que se espera la adquisición progresiva de la autonomía y el logro de un proyecto de vida personal (Rice, 1997), lo que se hace más evidente en población universitaria, la cual se encuentra en el proceso de lograr una profesión que permita la realización de dichas tareas de desarrollo.

Además, otros estudios en población universitaria encuentran de forma similar que las expectativas positivas frente al futuro son señaladas como la principal razón para vivir, lo cual es de suma relevancia como factor protector, si se toma en cuenta que los niveles de ideación 
suicida son más altos en esta edad que en otros períodos de la adultez (Koven, 2001).

La segunda razón para vivir en este grupo fue autoevaluación positiva, la cual implica la satisfacción que refiere la persona sobre su propia forma de ser. La tercera razón es creencias de afrontamiento, que consiste en la idea de que existen varias alternativas para enfrentar la adversidad sin la necesidad de pensar en el suicidio. Esto último se relaciona con lo planteado por Koven (2001), quien halló que se encuentran mayores niveles de creencias en el afrontamiento exitoso de las situaciones difíciles durante la adultez temprana y tardía, mientras que esas creencias decaen durante la adultez media.

Finalmente se encuentran los aspectos interpersonales, relaciones con pares y relaciones familiares, que se asocian con la vivencia de contar con amigos y familiares capaces de brindar el apoyo necesario.

Es interesante observar las relaciones establecidas entre las áreas de la prueba. Existe una correlación mediana y estadísticamente significativa entre expectativas hacia el futuro y creencias de afrontamiento. Este hallazgo apoya lo planteado por Dogra et al. (2011), quienes señalan que se cuenta con una visión positiva del futuro, es decir, se tiene confianza en que las metas personales se realizarán, en mayor medida cuando se tiene confianza en la capacidad para afrontar los problemas. Por otro lado, de manera coherente se halla una correlación mediana y estadísticamente significativa entre las áreas relaciones de pares y relaciones familiares, lo cual es coherente con la noción de que el establecimiento de la intimidad en la adultez temprana consiste en la posibilidad del establecimiento de relaciones interpersonales satisfactorias con amigos, pareja y familia. Esto se diferencia del tipo de vínculos establecidos en el estadio anterior, la adolescencia, centrada en las relaciones de pares.

Para la validez convergente se analizaron las relaciones entre el RFL-YA y las medidas de esperanza (HHS) y de sentido de coherencia (SOC). Las correlaciones halladas van en el mismo sentido que los estudios de Chimich y Nekolaichuk (2004), quienes encontraron que en pacientes deprimidos los sentidos de coherencia y esperanza se hallan disminuidos, lo que los llevó a plantear que ambas variables son 
factores protectores contra las situaciones estresantes en general, y contra la enfermedad depresiva y el suicidio de forma específica.

En nuestra investigación se halla una relación altamente significativa entre la medida de sentido de coherencia y las razones para vivir, que apoyaría los planteamientos de Edwards y Holden (2003). Estos autores encontraron, en 298 estudiantes universitarios, que el sentido de coherencia contribuye de forma significativa a la predicción de la ideación suicida en tanto se fundamenta en las creencias en la capacidad para el afrontamiento con la que cuenta una persona.

Las correlaciones altas y significativas entre la medida de esperanza (HHS) y las razones para vivir se fundamentan en el hecho de que niveles elevados de esperanza disminuyen el riesgo de sintomatología depresiva (Arnau, Rosen, Finch, Rhudy \& Fortunato, 2007; Chen \& Chen, 2008; Mascaro \& Rosen, 2005) e ideación suicida (Vincent, Boddana \& MacLeod, 2004; Wang et al., 2007). La relación hallada entre esperanza y razones para vivir apoyaría lo planteado por Dogra, Basu y Das $(2008,2011)$, quienes postulan que estas últimas promueven un sentido de esperanza a la vida y, por tanto, reducen la posibilidad de ideación y de realización de la conducta suicida.

De esta manera, esta investigación demuestra el valor psicométrico de la escala Razones para Vivir en Jóvenes Adultos (RFL-YA), en tanto presenta buenos indicadores en términos de confiabilidad y validez. Rescatamos que este instrumento favorezca una evaluación más completa del riesgo suicida al brindar información sobre las razones para vivir reportadas por el propio individuo. Esto resulta central para el desarrollo de proyectos de prevención en salud mental que identifiquen de forma temprana casos de riesgo.

Asimismo, encontramos que identificar las razones para vivir puede ser una herramienta útil para la intervención clínica, ya que facilita el acceso a los recursos en los cuales se puede apoyar un paciente. Además, explicitar dichas razones puede tener importantes efectos terapéuticos en tanto permite que el paciente se focalice en argumentos positivos para vivir y se aleje de pensamientos negativos persistentes, presentes en la depresión e ideación suicida. Por tanto, la explicitación de las 
razones para vivir ayuda en sí mismo al cambio terapéutico (Wang et al., 2007). Esto es consistente con las investigaciones que hallan que el simple hecho de verbalizar y/o escribir argumentos positivos para la vida y anticiparse ante los retos de la misma manera incrementa los niveles de bienestar y afectividad positiva, contribuyendo a disminuir la sintomatología depresiva y sus riesgos (Barra, 2003; Larsen \& Prizmic, 2008; Niederhoffer \& Pennebaker, 2009).

\section{Referencias}

Antonovsky, A. (1993). The structure and properties of the Sense of Coherence Scale. Social Science and Medicine, 36, 725-733.

Arévalo, M. (2007). Stress and extreme poverty in Peruvian women. Nijmegen, Holanda: Radbout Universiteit Nijmegen.

Arnau, R., Rosen, D., Finch, J., Rhudy, J. \& Fortunato, V. (2007). Longitudinal effects of hope on depression and anxiety: A latent variable analysis. Journal of Personality, 75(1), 43-63.

Arnau, R., Martínez, P., Niño de Guzmán, I., Herth, K. \& Yoshiyuki, C. (2010). A Spanish-language version of the Herth Hope Scale. Educational and Psychological Measurement, 70(5), 808-824.

Barra, E. (2003). Psicología de la salud. Santiago de Chile: Mediterráneo. Britton, P., Duberstein, P., Conner, K., Heisel, M., Hirsch, J. \& Conwell, Y. (2008). Reasons for living, hopelessness, and suicide ideation among depressed adults 50 years or older. The American Journal of Geriatric Psychiatry, 16(9), 736-741.

Chen, H. \& Chen, J. (2008). Structural equation modeling: Hope, coping styles and emotions of impoverished undergraduates. Chinese Journal of Clinical Psychology, 16(4), 392-394.

Chimich, W. \& Nekolaichuk, C. (2004). Exploring the links between depression, integrity, and hope in the elderly. Canadian Journal of Psychiatry, 49(7), 428-433.

Dogra, A., Basu, S. \& Das, S. (2008). The roles of personality, stressful life events, meaning in life, reasons for living on suicidal ideation: 
A study in college students. SIS Journal of Projective Psychology and Mental Health, 15, 52-57.

Dogra, A., Basu, S. \& Das, S. (2011). Impact of meaning of life and reasons for living to hope and suicidal ideation: A study among college students. SIS Journal of Projective Psychology and Mental Health, 18, 89-102.

Edelstein, B., Heisel, M., McKee, D., Martin, R., Koven, L. \& Duberstein, P. (2009). Development and psychometric evaluation of the reasons for living-older adult scale: A Suicide Risk Assessment Inventory. Gerontologist, 49(6), 736-745.

Edwards, M. \& Holden, R. (2003). Coping, meaning in life, and suicidal manifestations: Examining gender differences. Journal of Clinical Psychology, 59(10), 1133-1150.

George, D. \& Mallery, P. (2001). SPSS for Windows step by step. A simple guide and reference (3ra. ed.). Nueva York: Allyn \& Bacon.

Gutiérrez, P., Osman, A., Barrios, F., Kopper, B., Baker, M. \& Haraburda, C. (2002). Development of the Reasons for Living Inventory for Young Adults. Journal of Clinical Psychology, 58(4), 339-357.

Instituto Nacional de Salud Mental "Honorio Delgado Hideyo Noguchi”. (2002). Estudio epidemiológico metropolitano en salud mental 2002. Anales de Salud Mental, 18(1 y 2).

Jong, P. (2004). Testing the test. Groningen, Holanda: Universidad de Groningen.

Koven, L. (2001). Reasons for living across the lifespan (Tesis de maestría, West Virginia University, Estados Unidos). De la base de datos Dissertations and Theses: A\&I.

Larsen, R. \& Prizmic, Z. (2008). The regulation of emotional wellbeing: Overcoming the hedonic treadmill. En M. Eid \& R. Larsen (Eds.), The science of subjective well-being (pp. 258-289). Nueva York: Guilford Press.

Linehan, M., Goodstein, J., Nielsen, S. \& Chiles, J. (1983). Reason for staying alive when you are thinking of killing yourself: The Reason for Living Inventory. Journal of Consulting and Clinical Psychology, 51(2), 276-286. 
Martínez, P., Cassaretto, M. \& Herth, K. (2012, en prensa). Propiedades psicométricas de la Escala de Esperanza de Herth en español. Revista Iberoamericana de Diagnóstico y Evaluación Psicológica.

Mascaro, N. \& Rosen, D. (2005). Existential meaning's role in the enhancement of hope and prevention of depressive symptoms. Journal of Personality, 73(4), 985-1013.

Muehlenkamp, J., Gutiérrez, P., Osman, A. \& Barrios, F. (2005). Validation of the Positive and Negative Suicide Ideation (PANSI) Inventory in a diverse sample of young adults. Journal of Clinical Psychology, 61(4), 431-445.

Niederhoffer, K. \& Pennebaker, J. (2009). Sharing one's story: On the benefits of writing or talking about emotional experience. En C. Snyder, J. Shane \& S. López (Eds.), Oxford handbook of positive psychology. Nueva York: Oxford University Press.

Oquendo, M., Baca-García, E., Graver, R., Morales, M., Montalvan, V. \& Mann, J. (2000). Spanish adaptation of the Reasons for Living Inventory. Hispanic Journal of Behavioral Sciences, 22(3), 369-381.

Organización Mundial de la Salud. (2001). World Health Report 2001. Mental health: New understanding, new hope. Ginebra, Suiza: Autor. Osman, A., Downs, W., Kopper, B., Barrios, F., Baker, M., Osman, J. et al. (1998). The Reasons for Living Inventory for Adolescents (RFL-A): Development and psychometric properties. Journal of Clinical Psychology, 54(8), 1063-1078.

Paredes, C., Orbegoso, O. \& Rosales, P. (2006). Caracterización de pacientes con intento de suicidio en un hospital general de Lima, 1995-2004. Revista Peruana de Medicina Experimental y Salud Pública, 23(4), 293-296.

Rice, F. P. (1997). Desarrollo humano (2da. ed.). México D. F.: PrenticeHall.

Rosero, M. (2008). Propiedades psicométricas de una versión adaptada y una ampliada del Inventario de Razones para Vivir en Adultos Jóvenes (RFL-YA, Gutiérrez et al., 2002), en una muestra de estudiantes universitarios de la ciudad de San Juan de Pasto (Tesis de licenciatura inédita). Universidad de Nariño, Colombia. 
Saravia, J. C. (2010). Sentido de coherencia en pacientes limitrofes y estudiantes de ingeniería (Tesis inédita). Pontificia Universidad Católica del Perú, Lima.

Valencia, J. et al. (2009). Validación del Inventario de Razones para Vivir (RFL) en sujetos con conducta suicida de Colombia. Revista Colombiana de Psiquiatría, 38(1), 67-84.

Vincent, P., Boddana, P. \& MacLeod, A. (2004). Positive life goals and plans in parasuicide. Clinical Psychology \& Psychotherapy, 11, 90-99.

Von Bothmer, M. \& Fridlund, B. (2003). Self-rated health among university students in relation to sense of coherence and other personality traits. Scandinavian Journal of Caring Sciences, 17(4), 347-357.

Wang, M., Lightsey, O., Pietruszka, T., Uruk, A. \& Wells, A. (2007). Purpose in life and reasons for living as mediators of the relationship between stress, coping, and suicidal behavior. The Journal of Positive Psychology, 2(3), 195-204.

Westefeld, J., Range, L., Rogers, J., Maples, M., Bromley, J. \& Alcorn, J. (2000). Suicide: An overview. The Counseling Psychologist, 28(4), 445-510.

Recibido: 20 de setiembre, 2011

Aceptado: 20 de enero, 2012 